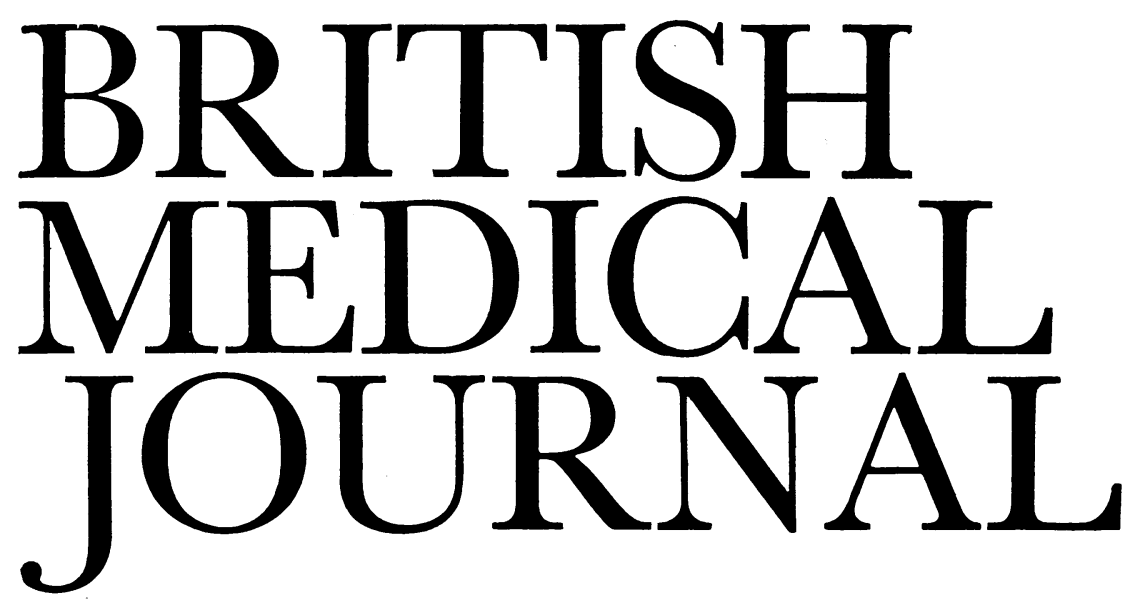

LONDON, SATURDAY 16 JULY 1983

\title{
Pruritus ani
}

Itching or irritation of the perianal skin is a disorder partly dermatological, partly psychological, and occasionally proctological. Because of the precision of its fundamental location the sufferer is frequently referred to a proctologist when simple or homely treatments fail.

Perianal dermatitis is a cross between nappy rash, athlete's foot, and a self inflicted injury. In most patients the problem is due either to inadequate cleansing of the anus or to overvigorous attempts to polish it clean.

A baby's bottom has long been a yardstick of smoothness; as many mothers ruefully observe, it is remarkably easy to clean a baby's bottom after a normal formed stool has been passed; one wipe with soft tissue usually suffices. The mother herself may not be so happy if her own perianal area bears external dermal evidence of the piles of pregnancy, possibly aggravated by irregularities associated with imperfect suturing of episiotomy wounds. The grandmother is often even less fortunate: her anal skin is furrowed with the ravages of time, and her aging anal sphincter is often inadequate to prevent leakage of mucus of even faeces.

The older generations know that if they leave a young baby with its perineum in contact with faeces the skin will very soon -become raw and excoriated. Perhaps they do not realise that if they leave minute particles of faecal material in the crevices of their own irregular anal skin, they too will be prone to adult nappy rash.

I do not mean to imply that pruritus or the lack of anal cleanliness is a female prerogative. It is a truly unisex affliction -for though men do not suffer from episiotomy, parturition, or damage to pudendal nerves they tend to be more prone to addiction to hot curries and alcoholic excess and are perhaps the more likely of the sexes to be obsessional perianal polishers.

Once the sensitive skin of the perianal area has been damaged a vicious circle begins. Because of the rich cutaneous nerve supply the damaged skin is sensitive and either itches or is painful. The sufferer then tends to scratch the affected area so damaging the epithelium further. Once the natural resistance of the perianal skin to infection is damaged saprophitic bacteria and fungi from the skin can gain access, giving rise to a dermatitis. He may then develop a sensitivity to the byproducts of micro-organisms and, particularly if he has an eczematoid tendency, a skin sensitivity reaction may twist the vicious circle into a vicious spiral.

At this stage the sufferer may qualify for the title of "a patient" and become subject to even greater hazards-those of perianal polypharmacy. The pharmaceutical industry (and often the medical profession) will encourage the patient to use compounds apparently specially prepared for the problem and marketed with appropriately enticing names. These balms usually contain steroids, local anaesthetics, and antibiotics and are often prepared as ointments in a greasy base. It would be difficult to design any combination of drugs more likely to produce skin sensitivity, particularly in susceptible people. Topical steroids applied for long tend to damage the natural resistance of the skin to fungal infection, and topical antibiotics and anaesthetic agents are particularly prone to induce skin sensitivity. Some of the worst cases of perianal dermatitis that present to the dermatologist or proctologist are the result of using these pharmaceutical preparations.

The first line of treatment for severe perianal dermatitis is to stop all medication. That the preparations work at all is, perhaps, surprising but they do; the local anaesthetic in particular may help the patient through the worst symptoms of what can be a naturally remitting disorder. It is the prolonged use of the potentially allergenic substances that is particularly dangerous, so that if they are used at all treatment should be limited to a maximum of one week.

Predisposing tendencies to pruritus ani include any one of a variety of conditions that will lead to perpetually moist perianal skin. These include vaginal discharge, urinary leakage, excessive sweating, mucous discharge from the anus with third degree piles or mucosal prolapse, a purulent discharge associated with a fistula in ano or perianal sepsis, and frequent defecation-particularly of watery stools. Moisture in the natal cleft may be aggravated by wearing tight clothes that compress the buttocks, underclothes of synthetic non-porous material, a hot occupation, or prolonged sitting on plastic seats. The increasing popularity of nylon tights and nylon underpants in recent decades has been said to be associated with a real increase in the incidence of perineal pruritus.

Other predisposing factors include systemic diseases such as diabetes, intestinal helminthiasis, scabies, anogenital herpes, molluscum contagiosum, a tendency to an eczematoid skin reaction, and a tendency to fungal skin infection elsewhere on the body. With such a formidable list of predisposing factors it seems surprising that anyone escapes this modern scourgeand I believe few do.

The essentials of treatment are keeping the perianal skin 
scrupulously clean, dry, and protected from physical trauma. Anal cleanliness has been a preoccupation of man from time immemorial. The art is practised with greater care, cunning, and tradition in tropical and subtropical climes than it is in Britain. I have heard an eminent American physiologist observe that the reason why most Englishmen looked so miserable was because they had perianal soreness from their habit of using tracing paper for postdefecation cleansing, whereas the French were a happier looking race because of their almost universal use of the bidet. Certainly the French have long been aware of the evil consequences of poor perianal hygiene. The notorious François Rabelais wrote a verse in about 1520 :

St Anthony's fire sieze on thy toane,

If thy Dirty Dounby Thou dost not wipe, ere thou be gone.

If, then, perianal cleanliness is a prerequisite to the control of pruritus ani cleanliness should be achieved with the minimum of trauma. Dry wiping of the skin is much more likely to traumatise than wiping with wet material; ideally one should wash gently with a jet of water, a moist cloth, or cotton wool. Wet wiping of the perianal skin is also much more efficient than dry wiping. The truth of this statement can be observed quite simply by sufferers and non-sufferers alike. If the anus is cleaned efficiently and gently with soft dry tissues and then when apparently clean it is wiped again using moist tissue such as "Wet Ones" or "Baby Wipes" most adults will observe that a recognisable amount of faecal material is removed after apparent complete cleanliness with a dry wipe. Once this observation has been shown to sufferers from pruritus ani they will easily be persuaded always to carry sachets or drums of wet wipes with them when they travel and particularly if they have to defecate away from home and washing facilities. This is particularly important in those people who habitually defecate after they arrive at work in the morning.

Conditions which predispose to faecal leakage make anal cleanliness particularly difficult. The elderly, particularly women, and women who have suffered damage from parturition are most likely to have minor or major degrees of incontinence. Is it ever worth while trying to improve their continence in the management of pruritus ani ? Certainly those in whom the incontinence is in itself a major symptom require treatment. The specialist has at his command measures designed to improve the function of the anal sphincter such as physiotherapy with exercises or faradic stimulation and the use of portable self treatment devices with anal sphincter stimulators. Anecdotal evidence suggests that these sometimes relieve symptoms in patients with pruritus ani, but scientific proof from prospective trials is lacking. I am sure that measures to improve the power of the anal sphincter are much less important in the management of pruritus than attention to hygiene. Nevertheless, patients with true incontinence who can be cured or improved are often delighted to have their anal irritation relieved. On the other hand I do not believe that surgical intervention (such as a postanal repair of the pelvic floor) is indicated simply to relieve pruritus.

Many patients with pruritus ani are regular imbibers of aperients and this often contributes to their problems. Habitués of liquid paraffin may have a perpetual slight oil leak, while cathartic addicts and bitter beer devotees probably deserve their perianal soreness. I believe-and much of this art is belief rather than science-that a high bran intake is bad for patients with pruritus ani, possibly because of the stool frequency it induces, but possibly also because the minute flakes of bran are particularly difficult to cleanse from the perianal crevices. Nevertheless, in defence of bran fans it must be said that bran makes many people into the satisfied deliverers of what we might call the "normal stool." I remember one of my mentors defining this wholesome objective as "twice round the pan and pointed at both ends." Such happy defecators are rarely troubled by pruritus.

A dry perianal region is next in importance. Sufferers should avoid non-porous clothing and any garments that keep the buttocks held tightly together. Nudism is ideal but, sadly, often impracticable. I have no evidence to confirm or deny the $\stackrel{\vec{F}}{\rightarrow}$ belief that the strict adherence to traditional Scottish kilt wearing is a sure protection against pruritus ani.

To aid in the maintenance of a dry perianal region the local application of astringent lotions is particularly helpful when dermatitis produces skin exudation. The ancient Egyptians used infusions of acacia leaves for their perianal problems. $\overrightarrow{0}$ Possibly a more reliable but more expensive application is a $\overrightarrow{\vec{H}}$ $0.5 \%$ aqueous solution of silver nitrate. This readily oxidises in $\vec{\omega}$ light, however, and needs to be prepared fresh once or twice a $\frac{\sigma}{3}$ week; it also stains the fingers. Antifungal agents are useful if there is the characteristic "prairie fire" edge to the dermatitis indicating a fungal origin. Occasionally they may induce local irritation and sensitivity, so some dermatologists recommend $\mathbb{W}_{\infty}$ older, cheaper, less elegant lotions such as aqueous solution of pig magenta co.

Probably the single most effective measure in the control of 음 pruritus ani is the education of the patient in the nature of the $\vec{\overrightarrow{ }}$ condition and the rationale of its control. Like many busy $c$ proctologists I have prepared a printed handout with a list of $\stackrel{\varrho}{\gtrless}$ do's and don'ts. This allows the patient to refer to the $\vec{\varphi}$ instructions at leisure and to read it again whenever there is a recrudescence.

\section{J ALEXANDER-WILLIAMS}

Consultant Surgeon

General Hospital,

Birmingham B4 6NH

\section{Dyspnoea}

Difficulties in breathing comprise a variety of sensations which patients describe with an even greater variety of words. Merseysiders, remembering the days of the great transatlantic liners, talk about "blowing for tugs," while others complain of having to breathe too much, of not being able to breathe enough, of needing to take deep breaths, of gasping, suffocation, or tightness in the chest or throat. All these expressions have in common undue awareness of a function which is normally subconscious and of a discomfort which is not commensurate with the level of physical activity.

Probably no single mechanism or pathway is responsible for the sensation of dyspnoea. The classic experiments of Fowler ${ }^{1}$ and Campbell et $a l^{2}$ showed that the discomfort associated with holding the breath was not related to blood gas concentrations nor to stimuli from the lung but probably arose from receptors in the chest wall. Campbell and Howell ${ }^{3}$ postulated that the principal cause of dyspnoea was a mismatch between the tension set up in the respiratory muscles by stimuli from the brain and the resulting change in muscle length (length-tension inappropriateness). Vagal afferents may also contribute to the sensation of dyspnoea, ${ }^{4}$ perhaps from 